\title{
Inventory Model for Deteriorating Items with Stock Dependent Demand under the Effect of Inflation and Trade Credit Period
}

\author{
K. Prasanna Lakshmi \\ Assistant Professsor, \\ Department of Mathematics, Ethiraj College for \\ Women (Autonomous), Chennai, \\ Tamil Nadu, India
}

\author{
P. Parvathi \\ Principal, Rani Anna Government College for \\ Women, Tirunelveli, \\ Tamil Nadu, India
}

\begin{abstract}
In this study a deterministic inventory model for deteriorating items with stock dependent demand is developed. In this model the shortages are allowed and partially backlogged and the effect of inflation rate and delay in payments are discussed. This paper establishes an inventory model for the deteriorating items and stock dependent demand rate under inflation when the supplier offers a permissible delay to the purchaser. Then an optimal solution is obtained to find the relevant total optimal cost in two cases.
\end{abstract}

CASE-1: In this case, the length of the period with the positive inventory of items is longer than the credit period.

CASE-2: In this case, the permissible delay is longer than the length of the period with the positive inventory of items. The interest of purchasing cost is charged for the delay of payments by the retailers. In both the cases the total average inventory cost per unit time is minimized.

\section{Keywords}

Stock dependent demand, deteriorating items, inflation rate, trade credit period, shortages and backlogging

\section{INTRODUCTION}

Inventory plays a vital role in business to ensure smooth efficient running of its operation. Large number of research papers/Articles has been presented by many authors for controlling the inventory of deteriorating items such as volatile liquids, blood banks, medicines, fashion goods and non-deteriorating items such as wheat, rice, dry fruits etc. The control and the maintenance of inventories of deteriorating items with shortages have received much attention of several researchers in the recent years because most physical goods deteriorate overtime. In practice, the deterioration of items is a common phenomenon. Hence the impact of product deterioration should not be neglected in the decision process. The retailer must pay off as soon as the items are received. It is tacitly assumed in classical economic order quantity inventory model, a supplier frequently offers his retailers a delay of payment for settling the amount due. The permissible delay in payment is an effective method of attracting new customers and increasing sales. It may be applied as alternative to price discount because it does not provoke the competitors to reduce their prices and thus introduce lasting price reductions. Goyal (1) first considered the Economic Order Quantity Model under the conditions of permissible delay in payments. Hu and Liu (2) (2010) analyzed An Optimal replenishment policy for the EPQ model with permissible delay in payments and allowable shortages. Huang (3)

\begin{abstract}
(2007) developed Economic Order Quantity to order goods under permissible delay in payments. Chang, Wu and Chen (4) (2009) studied Optimal Payment Time with Deteriorating Items under the Inflation and Permissible Delay in Payments.
\end{abstract}

Trade credit period produces two benefits to the supplier: 1. It will attract the new customers who consider it to be a type of price reduction. 2 . It will cost the reduction in the sale outstanding since some established customer will order more and pay more promptly in order to take advantage of permissible delay more frequently. Chun-Tao Chang (5) developed An Inventory Model with Deteriorating Items for Constant Demand under Inflation and the Condition of Permissible Delay in Payments.

Inflation also plays an important role for the optimal order policy and influences the demand of certain products. The value of money goes down and erodes the future worth of saving and forces one for more current spending as inflation increases. The first Economic order quantity model by considering the effect of inflation was developed by Buza Cott(6) Suetal. He developed the model under the inflation of stocks dependent consumption rate and exponential decay. Most of the classical inventory model did not take into account the effects of inflation. This happened mostly because of the belief that the inflation would not influence the inventory policy variables to any significant rate. However most of the countries have suffered from large scale inflation and sharp decline in the purchasing power of money, the past few years. As a result the effect of inflation and time value of the money cannot be ignored for determining the optimal inventory policy.

Hou (7) studied An Inventory Model for Deteriorating Items with Stock Dependent Consumption Rate and Shortages under Inflation and Time Discounting. Hou and Lin(8) developed an EOQ Model for deteriorating items with price and stock dependent selling rates under inflation and time value of money. Mirzadesh, Syeed- Esfahani (9) analyzed An Inventory Model under Uncertain Inflationary Conditions, Final Production Rate and Inflation Dependent Rate for Deteriorating Items with Shortages.

Numerical examples and sensitivity analysis of parameters are given to illustrate the theoretical results.

\section{NOTATIONS}

$$
\begin{array}{ll}
\mathrm{H} & \text { The length of planning horizon } \\
\mathrm{R}(\mathrm{t}) & \text { Demand rate is deterministic(Stock }
\end{array}
$$

Dependent demand) 
The replenishment time interval

The inventory level at time ' $t$ ', $0 \leq t \leq$ $\mathrm{T}$

Constant rate of inflation per unit time $0 \leq \mathrm{i} \leq 1$

The unit purchasing cost at time ' $t$ ' where $\mathrm{P}$ is the unit purchasing cost at time zero.

The holding cost rate per unit time excluding interest charges.

Shortage cost, \$ per unit/year.

Opportunity cost due to lost sales \$ per unit.

The ordinary cost per order at time $t$ where $\mathrm{K}$ is the ordering cost at time zero.

The interest earned per \$ per year.

Interest charges which invested in inventory per $\$$ per year $I_{r} \geq I_{e}$.

Length of the replenishment cycle.

Time at which the shortage starts $0 \leq \mathrm{T}_{1} \leq \mathrm{T}$.

The order quantity.

The Constant rate of deterioration $0 \leq \theta \leq 1$.

number of replenishments during the period $\mathrm{H}[\mathrm{H}=\mathrm{nT}]$

TVC $\left(\mathrm{T}_{1}, \mathrm{~T}\right) \quad$ The Average total inventory cost per unit time.

$\mathrm{TVC}_{1}\left(\mathrm{~T}_{1}, \mathrm{~T}\right) \quad$ The average total inventory cost per unit time for $\mathrm{T}_{1}>\mathrm{M}$ in case (1).

$\mathrm{TVC}_{2}\left(\mathrm{~T}_{1}, \mathrm{~T}\right) \quad$ The average total inventory cost per unit time for $\mathrm{T}_{1}<\mathrm{M}$ In

case.(2)

\section{ASSUMPTIONS}

1. The demand rate functions $\mathrm{R}(\mathrm{t})$ is deterministic and is known as the function of the instantaneous stock level. The functional $R(t)$ is given by

$$
\mathrm{R}(\mathrm{t})=\left\{\begin{array}{c}
\alpha+\beta \mathrm{I}(\mathrm{t}) ; 0 \leq \mathrm{t}<\mathrm{T}_{1} ; \alpha>0 \\
\alpha ; \mathrm{T}_{1} \leq \mathrm{t}<\mathrm{T}
\end{array}\right\}
$$

2. Inflation rate is constant.

3. The replenishment rate is infinite.

4. There are no replacements or repair of deteriorated units.

5. Shortages are allowed and partially backlogged. The backlogged rate is defined to be $\frac{1}{1+\delta(T-t)}$.

When inventory is negative, the backlogging parameter $\delta$ is a positive constant.

6. During the trade credit period, if the account is not settled, the generated sales revenue is deposited in an interest bearing account. At the end of the permissible delay, the customer pays off all the units ordered and starts paying for the interest charges on the items in stock.

\section{MATHEMATICAL FORMULATION}

Due to the combined effects of demand and deterioration in the interval $[0, T]$ the level of inventory gradually decreases.
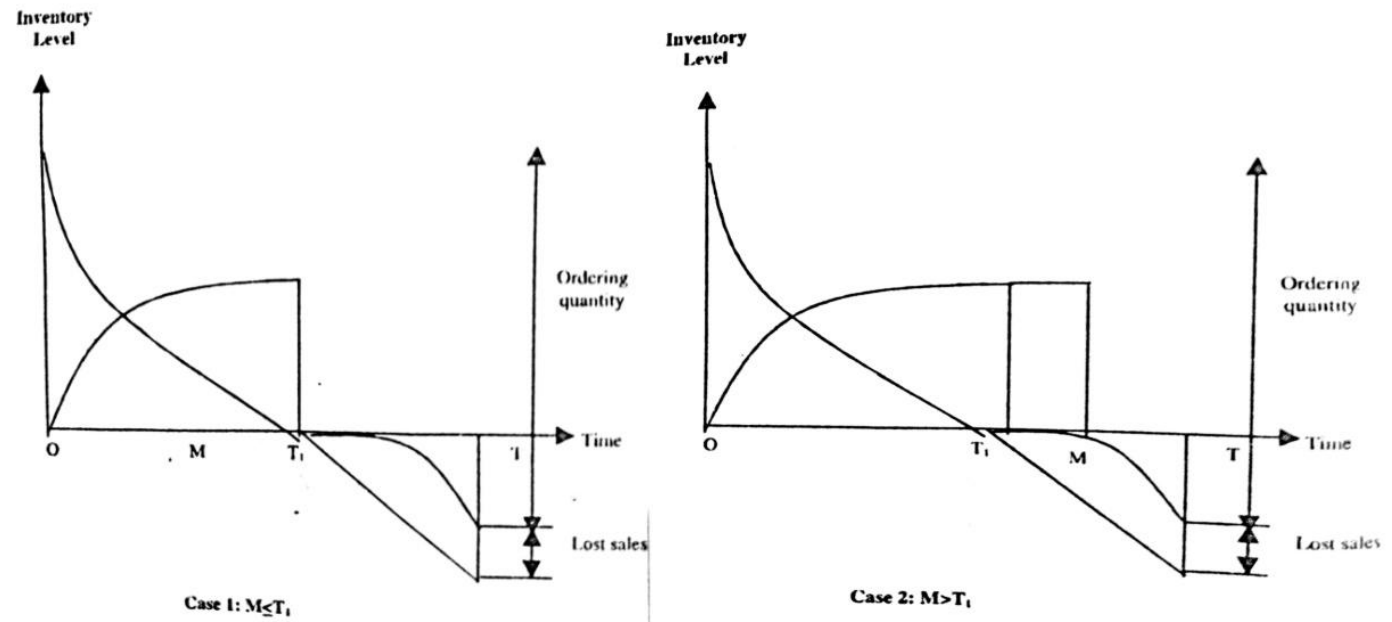

\section{Graphical Representation of Inventory System}

Hence the variation of inventory with respect to time can be described by the following differential equation.

$\frac{d I(t)}{d t}+\theta \mathrm{I}(\mathrm{t})=-\alpha-\beta I(t) \quad 0 \leq \mathrm{t} \leq \mathrm{T}_{1}$
With the boundary conditions :

$\mathrm{I}(0)=\mathrm{Q}$

$\mathrm{I}\left(\mathrm{T}_{1}\right)=0$ 
Solution of equation (I) is

$\mathrm{I}(\mathrm{t}) \mathrm{e}^{(\theta+\beta) \mathrm{t}}=-\alpha \int e^{(\theta+\beta) t} d t+C$

$$
=\frac{-\alpha e^{(\theta+\beta) t}}{(\theta+\beta)}+\mathrm{c}
$$

Using the boundary condition $\mathrm{I}\left(\mathrm{T}_{1}\right)=0, \mathrm{I}\left(\mathrm{T}_{1}\right) e^{(\theta+\beta) T_{1}}=$ $\frac{-\alpha e^{(\theta+\beta) T_{1}}}{(\theta+\beta)}+\mathrm{c}$

Therefore $\mathrm{C}=\frac{\alpha e^{(\theta+\beta) T_{1}}}{(\theta+\beta)}$

$\mathrm{I}\left(\mathrm{T}_{1}\right) e^{(\theta+\beta) t}=-\frac{\alpha e^{(\theta+\beta) t}}{(\theta+\beta)}+\frac{\alpha e^{(\theta+\beta) T_{1}}}{(\theta+\beta)}$

Dividing by $e^{(\theta+\beta) t}$,

$$
\begin{aligned}
\mathrm{I}(\mathrm{t}) & =\frac{-\alpha}{\theta+\beta)}+\frac{\alpha e^{(\theta+\beta)\left(T_{1}-t\right)}}{(\theta+\beta)} \\
& =\frac{\alpha}{\theta+\beta)}\left[e^{(\theta+\beta)\left(\left(T_{1}-t\right)\right.}-1\right] ; 0 \leq \mathrm{t}<\mathrm{T}_{1}
\end{aligned}
$$

Now by using the boundary conditions $\mathrm{I}(0)=\mathrm{Q}$ in the solution:

$\mathrm{Q}=\left[\frac{\propto}{\theta+\beta}\right]\left[e^{\left.(\theta+\beta) T_{1}\right)}-1\right]$

Consider $T_{1} \leq t<T$

$\frac{\mathrm{dI}(\mathrm{t})}{\mathrm{dt}}=\frac{-\propto}{1+\delta(T-t)}$

By solving the above equation

$$
I(t)=-\alpha \log [1+\delta(T-t)]\left(\frac{-1}{\delta}\right)+C
$$

Where $\mathrm{c}$ is the constant of integration.

Using the given boundary condition $I(t)$ as follows

$$
\begin{aligned}
& I(t)=-\frac{\alpha}{\delta}\left\{\log \left[1+\delta\left(T-T_{1}\right]-\log [1+\delta(T-t)]\right\}\right. \\
& \begin{aligned}
I(t)= & \\
\left(\frac{\alpha \theta-\beta}{\theta^{2}}\right) & {\left[e^{\theta\left(T_{1}-t\right)}-1\right]+} \\
& \frac{\beta}{\theta}\left[T_{1} e^{\theta\left(T_{1-t}-t\right]} \text { when } 0 \leq t<T_{1}\right.
\end{aligned} \\
& I(t)=-\frac{\alpha}{\delta}\left\{\log \left[1+\delta\left(T-T_{1}\right]-\log [1+\delta(T-t)]\right\}\right. \\
& \quad \text { when } T_{1} \leq t<T \ldots \ldots \ldots \ldots . . .(3)
\end{aligned}
$$

Since the length and time intervals are all the same,

$$
\begin{aligned}
& \mathrm{I}(\mathrm{jt}+\mathrm{T})= \\
& \frac{\alpha}{\theta+\beta}\left[e^{(\theta+\beta)\left(T_{1}-t\right)}-1\right] \\
& \text { for } 0 \leq j \leq n-1 ; 0 \leq t \leq T_{1} \\
& \frac{-\alpha}{\delta}\left\{\log \left(1+\delta\left(T-T_{1}\right)\right)-\log [1+\delta(T-t)]\right\} \\
& \text { for } T_{1} \leq t \leq T ; 0 \leq j \leq n-1 \text {. }
\end{aligned}
$$

The total relevant costs consists of
a. Cost of placing order.
b. Cost of purchasing.
c. Cost of carrying inventory excluding interest charges.

d. Shortage cost.

e. Opportunity cost.

f. The cost of interest charges for unsold items at the initial time or after permissible delay $\mathrm{M}$.

g. The interest earned from sales revenue during the permissible period.

Cost of placing order:

$$
\begin{aligned}
& \mathrm{K}(0)+\mathrm{K}(\mathrm{T})+\mathrm{K}(2 \mathrm{~T})+\ldots \ldots \ldots+\mathrm{K}(\mathrm{n}-1) \mathrm{T} \\
= & \mathrm{Ke}^{\mathrm{i} 0}+\mathrm{Ke}^{\mathrm{i} \mathrm{T}}+\mathrm{Ke}^{\mathrm{i} 2 \mathrm{~T}}+\ldots \ldots \ldots \ldots+\mathrm{Ke}^{\mathrm{i}(\mathrm{n}-1) \mathrm{T}} \\
= & \mathrm{K}\left[\frac{e^{i H}-1}{e^{i j}-1}\right]
\end{aligned}
$$

Cost of purchasing :-

$$
\begin{aligned}
& \mathrm{Q}\left[P e^{i o}+P e^{i T}+\ldots \ldots \ldots \ldots \ldots+P e^{i(n-1) T}\right] \\
= & {\left[\frac{P \alpha}{\theta+\beta}\right]\left[e^{(\theta+\beta) T}-1\right]\left[\frac{e^{i H}-1}{e^{i T}-1}\right] }
\end{aligned}
$$

Holding cost in the interval $[0, \mathrm{~T}]$ is( for one cycle)

$$
\begin{aligned}
\mathrm{Hc} & =\mathrm{h} \int_{0}^{T_{1}} I(t) d t \\
& =\mathrm{h} \int_{0}^{T_{1}} \frac{\alpha}{\theta+\beta}\left[e^{(\theta+\beta)\left(T_{1}-t\right)}-1\right] d t \\
& =\frac{\mathrm{h} \propto\left[\mathrm{e}^{(\theta+\beta) T_{1}}-1-\theta \mathrm{T}_{1}-\beta \mathrm{T}_{1}\right]}{(\theta+\beta)^{2}}
\end{aligned}
$$

Holding Cost For N Cycles Is

$$
\begin{aligned}
\mathrm{Hc} & =\mathrm{h} \sum_{j=0}^{n-1} P e^{i j t} \int_{0}^{T_{1}}[I(j T+t)] d t \\
\mathrm{Hc} & =\mathrm{h} \sum_{j=0}^{n-1} P e^{i j t} \int_{0}^{T_{1}}\left[e^{(\theta+\beta)\left(T_{1}-t\right)}-1\right] d t \\
& =\left[\frac{\mathrm{hP} \propto}{(\theta+\beta)^{2}}\right]\left[\mathrm{e}^{(\theta+\beta) \mathrm{T}_{1}}-(\theta+\beta) \mathrm{T}_{1}-1\right]\left[\frac{\mathrm{e}^{\mathrm{iH}}-1}{\mathrm{e}^{\mathrm{iT}}-1}\right]
\end{aligned}
$$

Deterioration cost in the interval $\left[0, T_{1}\right]$

$$
\begin{gathered}
=P \theta \int_{0}^{T_{1}} I(j T+t) d t \\
=P \theta \int_{0}^{T_{1}} \frac{\alpha}{(\theta+\beta)}\left[e^{(\theta+\beta)\left(T_{1}-t\right)}-1\right] \mathrm{dt} \\
=\frac{P \theta \propto\left[\mathrm{e}^{(\theta+\beta) \mathrm{T}_{1}}-1-\theta \mathrm{T}_{1}-\beta \mathrm{T}_{1}\right]}{(\theta+\beta)^{2}}
\end{gathered}
$$

Therefore for $\mathrm{n}$ cycles

$=\frac{\mathrm{P} \theta \alpha\left(\mathrm{e}^{(\theta+\beta) \mathrm{T}_{1}}-1-\theta \mathrm{T}_{1}-\beta \mathrm{T}_{1}\right)}{(\theta+\beta)^{2}}\left[\frac{\mathrm{e}^{\mathrm{iH}}-1}{\mathrm{e}^{\mathrm{iT}}-1}\right]$

During the stock out period two kinds costs to be considered. First to derive the shortage cost for the backlogged items and then to obtain the opportunity cost due to lost sales. The shortage cost over the period $\left[\mathrm{T}_{1}, \mathrm{~T}\right)$ denoted by $\mathrm{SC}$ is given by:

(i.e) Shortage is given by

$\mathrm{SC}=\mathrm{S} \int_{T_{1}}^{T} I(t) \mathrm{dt}$

substitute (4) in I(t)

$$
=\mathrm{S} \sum e^{i j t} \int_{T_{1}}^{T} \frac{-\alpha}{8}\left\{\operatorname { l o g } \left(1+\delta\left(T-T_{1}\right)-\log [1+\delta(T-\right.\right.
$$

t)]\} $\mathrm{dt}$

Therefore on simplification 


$$
\begin{aligned}
& \mathrm{SC}=\sum e^{i j t}\left(\frac{-S \propto}{8}\right)\left\{( T - T _ { 1 } ) \operatorname { l o g } \left(1+\delta\left(T-T_{1}\right)-\right.\right. \\
& \left(T-T_{1}\right) \log \left[1+\delta\left(T-T_{1}\right)\right]+\left(T-T_{1}\right) \frac{-1}{\delta} \log (1+ \\
& \left.\delta\left(T-T_{1}\right)\right\} \\
& \quad=-\sum e^{i j t} \frac{S \alpha}{\delta}\left\{\delta\left(T-T_{1}\right)-\log \left[1+\delta\left(T-T_{1}\right)\right]\right\}
\end{aligned}
$$

The cost cannot be negative, so the shortage cost is given for ' $n$ ' cycles:-

$$
\mathrm{Sc}=\frac{S \propto}{\delta^{2}}\left\{\delta\left(T-T_{1}\right)-\log \left[1+\delta\left(T-T_{1}\right)\right]\left[\frac{\mathrm{e}^{\mathrm{iH}}-1}{\mathrm{e}^{\mathrm{iT}}-1}\right]\right.
$$

Now the opportunity cost due to lost sales during the replenishments cycle denoted by OC is given by.

$$
\begin{aligned}
\mathrm{OC} & =\sum e^{i j t} \Pi \int_{T_{1}}^{T} \propto\left[1-\frac{1}{1+\delta(T-t)}\right] \mathrm{dt} \\
& =\sum e^{i j t} \frac{\Pi \propto}{\delta}\left\{\delta\left(T-T_{1}\right)-\log \left[1+\delta\left(T-T_{1}\right)\right]\right. \\
& =\frac{\Pi \propto}{\delta} \delta\left(T-T_{1}\right)-\log \left[1+\delta\left(T-T_{1}\right)\right]\left[\frac{\mathrm{e}^{\mathrm{iH}}-1}{\mathrm{e}^{\mathrm{iT}}-1}\right]
\end{aligned}
$$

Now consider $\mathrm{M}$ which is the permissible delay in settling the accounts offered by the supplier.

\section{Case $1: M \leq T_{1}$}

Since in this case the length of the period with positive inventory is longer than the credit period, the buyer can earn the interest with an annual rate $I_{e}$ in $\left[0, T_{1}\right)$.

Now the interest earned is denoted by $\mathrm{IE}_{1}$ is given by

$$
\begin{aligned}
& \mathrm{IE}_{1}=\mathrm{PI}_{\mathrm{e}} \sum_{j-0}^{n-1} e^{i j t} \int_{0}^{T_{1}}\left(T_{1}-t\right) R(t) d t \\
& \quad=\mathrm{PI}_{\mathrm{e}} \\
& . \sum_{j-0}^{n-1} e^{i j t} \int_{0}^{T_{1}}\left(T_{1}-t\right)\left[\alpha+\quad \beta \frac{\alpha}{\theta+\beta}\left[e^{(\theta+\beta)\left(T_{1}-t\right)}-\right.\right. \\
& 1]] \mathrm{dt}
\end{aligned}
$$

The interest charged in $(0, \mathrm{H})$ is after the fixed credit period. The buyer has to pay interest on the product still in stock with an annual rate $I_{r}: I_{p}$

$$
\begin{aligned}
& \text { Therefore } I_{p}=I_{r} \sum_{j=0}^{n-1} P e^{i j t} \int_{M}^{T_{1}} I(j T+t) d t \\
= & P I_{r} \sum_{j=0}^{n-1} e^{i j t} \int_{M}^{T_{1}} \frac{\alpha}{\theta+\beta}\left[e^{(\theta+\beta)\left(T_{1}-t\right)}-1\right] d t
\end{aligned}
$$

The total average cost in this case is

$$
\begin{aligned}
& \mathrm{TVC}_{1}=\frac{1}{T}\left[\mathrm{~K}+\mathrm{HC}+\mathrm{DC}+\mathrm{SC}+\mathrm{OC}+\mathrm{IP}-\mathrm{IE}_{1}\right] \\
& =\frac{1}{T}\left[K+\frac{h p \alpha}{(\theta+\beta)^{2}}\left(e^{(\theta+\beta) T_{1}}-(\theta+\beta) T_{1}-1\right)\right]+ \\
& P \theta \alpha \frac{\left[e^{(\theta+\beta) T_{1}}-1-\theta T_{1}-\beta T_{1}\right]}{(\theta+\beta)^{2}}+\frac{S \alpha}{\delta^{2}}\left\{\delta\left(T-T_{1}\right)-\log [1+\right. \\
& \left.\left.\delta\left(T-T_{1}\right)\right]\right\}+\frac{\pi \alpha}{\delta}\left\{\left\{\delta\left(T-T_{1}\right)-\log \left[1+\delta\left(T-T_{1}\right)\right]\right\}\right\} \\
& \left.+I P_{1}-I E_{1}\right]\left[\frac{\mathrm{e}^{\mathrm{iH}}-1}{\mathrm{e}^{\mathrm{iT}}-1}\right]
\end{aligned}
$$

The total average cost per unit time can be minimized on the optimal values of $\mathrm{T}_{1}$ and $\mathrm{T}$ i.e $\left(T_{1}^{*} \& T^{*}\right)$ can be found by solving the following equations.

$$
\frac{\partial \mathrm{TVC}_{1}(\mathrm{~T}, \mathrm{~T})}{\partial \mathrm{T}_{1}}=0---(\mathrm{A}) \quad ; \quad \frac{\partial \mathrm{TVC}_{1}(\mathrm{~T}, \mathrm{~T})}{\partial \mathrm{T}}=0-----(\mathrm{B})
$$

Provided they satisfy the sufficient conditions:

$$
\left[\frac{\partial^{2} \mathrm{TVC}_{1}\left(\mathrm{~T}_{1}, \mathrm{~T}\right)}{\partial \mathrm{T}_{1}^{2}}\right]>0
$$

$$
\begin{gathered}
{\left[\frac{\partial^{2} \mathrm{TVC}_{1}\left(\mathrm{~T}_{1}, \mathrm{~T}\right)}{\partial \mathrm{T}^{2}}\right]>0} \\
{\left[\left[\frac{\partial^{2} \mathrm{TVC}_{1}\left(\mathrm{~T}_{1}, \mathrm{~T}\right)}{\partial \mathrm{T}_{1}{ }^{2}}\right]\left[\frac{\partial^{2} \mathrm{TVC}_{1}\left(\mathrm{~T}_{1} \mathrm{~T}\right)}{\partial \mathrm{T}^{2}}\right]-\left[\frac{\partial^{2} \mathrm{TVC}_{1}\left(\mathrm{~T}_{1}, \mathrm{~T}\right)}{\partial \mathrm{T}_{1} \partial \mathrm{T}}\right]^{2}\right]>0}
\end{gathered}
$$

To find the optimal values of $\mathrm{T}_{1}$ and $\mathrm{T}$ use the following algorithm

\section{Algorithm:}

Step-1 :perform(1) - (4)

1. Start with $\mathrm{T}_{1}(1)=\mathrm{M}$.

2. Substituting $\mathrm{T}_{1}$ into equation (A) and find $\mathrm{T}_{(1)}$.

3. Using $\mathrm{T}_{(1)}$ find $\mathrm{T}_{(2)}$ form equation.(B).

4. Repeat (2) and (3) until no change occurs in the value of $\mathrm{T}_{1}$ and $\mathrm{T}$.

Step -2: Compare $\mathrm{T}_{1}$ and $\mathrm{M}$

(i) If $\mathrm{M} \leq \mathrm{T}_{1}, \mathrm{~T}_{1}$ is feasible then go to step 3 .

(ii) If $\mathrm{M}>\mathrm{T}_{1}, \mathrm{~T}_{1}$ is not feasible, set $\mathrm{T}_{1}=\mathrm{M}$ and find the corresponding value of $\mathrm{T}$ from (B) then go to step 3 .

\section{Step-3:}

Calculate corresponding TVC,$\left(\mathrm{T}_{1}{ }^{*}, \mathrm{~T}^{*}\right)$

\section{Case 2: $M>T_{1}$}

Since $\mathrm{T}_{1}<\mathrm{M}$ the buyer earns the interest during the period $(\mathrm{O}, \mathrm{M})$ and pays no interest.

The interest earned in this case is denoted by $\mathrm{IE}_{2}$ is given by

$\mathrm{IE}_{2}=\sum_{0}^{n-1} e^{i j T} \mathrm{P}$. Ie $\left\{\int_{0}^{T_{1}}(M-t) R(t) d t\right.$

$[R(t)$ is from (1)]

$$
=\sum_{0}^{n-1} e^{i j T} \mathrm{P} \text {. Ie } \int_{0}^{T_{1}}\left(M-T_{1}+T_{1}-t\right)(\alpha+
$$

$\beta I(t)) d t)$

$$
\begin{gathered}
=\sum_{0}^{n-1} e^{i j T} \text { P.Ie } \\
\int_{0}^{T_{1}}\left(T_{1}-t\right)\left[\alpha+\beta\left(\frac{\alpha}{\theta+\beta}\right)\left\{e^{(\theta+\beta)\left(T_{1}-t\right)}-1\right\}\right] d t+ \\
\sum_{0}^{n-1} e^{i j T .} \text { P.Ie } \cdot \int_{0}^{T_{1}}\left(M-T_{1}\right) \\
{\left[\alpha+\beta\left(\frac{\alpha}{\theta+\beta}\right)\left\{e^{(\theta+\beta)\left(T_{1}-t\right)}-1\right\}\right] d t}
\end{gathered}
$$

Therefore the total average cost in the case is

$$
\begin{aligned}
& \mathrm{TVC}_{2}=\frac{\mathrm{K}+\mathrm{HC}+\mathrm{DC}+\mathrm{SC}+\mathrm{OC}-\mathrm{IE}_{2}}{\mathrm{~T}} \\
& \quad=\frac{1}{T}\left(K+\frac{h p \alpha}{(\theta+\beta)^{2}}\left(e^{(\theta+\beta) T_{1}}-(\theta+\beta) T_{1}-1\right)+\right. \\
& P \theta \alpha \frac{\left[e^{\left.(\theta+\beta) T_{1}-\theta T_{1}-\beta T_{1}-1\right]}\right.}{(\theta+\beta)^{2}}+\frac{S \alpha}{\delta^{2}}\left\{\delta\left(T-T_{1}\right)-\log [1+\right. \\
& \left.\left.\delta\left(T-T_{1}\right)\right]\right\}+\frac{\pi \alpha}{\delta} \delta\left(T-T_{1}\right)-\log \left[1+\delta\left(T-T_{1}\right)\right] \\
& \left.-I E_{2}\right)\left[\frac{\mathrm{e}^{\mathrm{iH}}-1}{\mathrm{e}^{\mathrm{iT}}-1}\right]
\end{aligned}
$$

For minimising the total average inventory cost per unit time, it is necessary to find the optimal value of $T_{1}$ and $T$ which are the solutions of the following equations

$$
\begin{gathered}
\frac{\partial T V C_{2}\left(T_{1}, T\right)}{\partial T_{1}}=0 \quad------(\mathrm{C}) \\
\frac{\partial T V C_{2}\left(T_{1}, T\right)}{\partial T}=0
\end{gathered}
$$


$\left[\frac{\partial^{2} \mathrm{TVC}_{1}(\mathrm{~T}, \mathrm{~T})}{\partial \mathrm{T}_{1}^{2}}\right]>0 ;\left[\frac{\partial^{2} \mathrm{TVC}_{1}(\mathrm{~T}, \mathrm{~T})}{\partial \mathrm{T}^{2}}\right]>0$

$\left[\left[\frac{\partial^{2} \mathrm{TVC}_{2}(\mathrm{~T}, \mathrm{~T})}{\partial \mathrm{T}_{1}^{2}}\right]\left[\frac{\partial^{2} \mathrm{TVC}_{2}\left(\mathrm{~T}_{1}, \mathrm{~T}\right)}{\partial \mathrm{T}^{2}}\right]-\left[\frac{\partial^{2} \mathrm{TVC}_{2}\left(\mathrm{~T}_{1}, \mathrm{~T}\right)}{\partial \mathrm{T}_{1} \partial \mathrm{T}}\right]^{2}\right]>0$

To find the optimal values of $\mathrm{T}_{1}$ and $\mathrm{T}$ use the following algorithm.

\section{Algorithm:}

Step-1: perform(1) - (4)

5. Start with $\mathrm{T}_{1}(1)=\mathrm{M}$.

6. Substituting $\mathrm{T}_{1}$ into equation $(\mathrm{C})$ and find $\mathrm{T}_{(1)}$.

7. Using $\mathrm{T}_{(1)}$ find $\mathrm{T}_{(2)}$ form equation.(D).
8. Repeat (2) an d (3) until no change occurs in the value of $\mathrm{T}_{1}$ and $\mathrm{T}$.

Step -2: Compare $\mathrm{T}_{1}$ and $\mathrm{M}$

(i) If $\mathrm{M} \leq \mathrm{T}_{1}, \mathrm{~T}_{1}$ is feasible then go to step 3 .

(ii) If $M>T_{1}, T_{1}$ is not feasible, set $T_{1}=M$ and find the corresponding value of $\mathrm{T}$ from (D) then go to step 3 .

\section{Step-3:}

Calculate corresponding $\mathrm{TVC}_{2}\left(\mathrm{~T}_{1}{ }^{*}, \mathrm{~T}^{*}\right)$

\section{NUMERICAL EXAMPLES}

\section{Example-1}

Let $\mathrm{H}=1$ year , $\alpha=1000, \beta=0.3 ; \mathrm{h}=1.2, \mathrm{P}=20, \mathrm{k}=100 ; \mathrm{S}=30 ; \delta=1 ; \mathrm{I}_{\mathrm{r}}=0.15, \mathrm{I}_{\mathrm{e}}=0.13 ; \theta=0.08, \mathrm{M}=2 / 365 ; \mathrm{i}=0.03 ; \pi=15$; $\mathrm{n}=2$

From the table 1, if $\mathrm{K}$ increases from 100 to 250, the total average cost increases. As $\mathrm{K}$ increases the total average cost also increases

Table1: Sensitivity analysis on $\mathrm{K}$

\begin{tabular}{|c|c|c|c|c|c|c|c|}
\hline \multirow{2}{*}{$\mathrm{K}$} & \multicolumn{3}{|c|}{ CASE-I } & \multicolumn{3}{c|}{ CASE-II } & TVC \\
\cline { 2 - 7 } & $\mathrm{T}^{*}$ & $\mathrm{~T}_{1}{ }^{*}$ & $\mathrm{TVC}_{1}$ & $\mathrm{~T}^{*}$ & $\mathrm{~T}_{1}{ }^{*}$ & $\mathrm{TVC}_{2}$ & $\begin{array}{c}\left.\text { Min }_{\left(\mathrm{TVC}_{1},\right.} \mathrm{TVC}_{2}\right) \\
\end{array}$ \\
\hline 100 & 0.1100 & 0.0707 & 3620.24 & 0.1071 & 0.0660 & 3718.26 & 3620.24 \\
\hline 150 & 0.1346 & 0.0857 & 4440.84 & 0.1310 & 0.0807 & 4561.14 & 4440.84 \\
\hline 200 & 0.1554 & 0.0989 & 5133.41 & 0.1512 & 0.0932 & 5272.64 & 5133.41 \\
\hline 250 & 0.1737 & 0.1105 & 5744.14 & 0.1690 & 0.1041 & 5900.18 & 5744.14 \\
\hline
\end{tabular}

\section{Example 2:}

Let $\mathrm{H}=1$ year , $\alpha=1000, \beta=0.3 ; \mathrm{h}=1.2, \mathrm{P}=20, \mathrm{k}=200 ; \mathrm{S}=30 ; \delta=1 ; \mathrm{I}_{\mathrm{r}}=0.15, \mathrm{I}_{\mathrm{e}}=0.13$;

$\theta=0.08, \mathrm{M}=2 / 365 ; \mathrm{i}=0.03 ; \pi=15 ; \mathrm{n}=2$

From the table 2, if $\mathrm{M}$ increases from 100 to 250 , the total average cost decreases.

Table 2: Sensitivity analysis for $M$ :

\begin{tabular}{|c|c|c|c|c|c|c|c|}
\hline \multirow{2}{*}{$\mathrm{M}$} & \multicolumn{3}{|c|}{ CASE I } & \multicolumn{3}{c|}{ CASE II } & TVC \\
\cline { 2 - 7 } & $\mathrm{T}^{*}$ & $\mathrm{~T}_{1}{ }^{*}$ & $\mathrm{TVC}_{1}$ & $\mathrm{~T}^{*}$ & $\mathrm{~T}_{1}{ }^{*}$ & $\mathrm{TVC}_{2}$ & $\begin{array}{c}\text { Min ( } \\
\mathrm{TVC}_{1,} \\
\mathrm{TVC}_{2}\end{array}$ \\
\hline 5 & 0.1555 & 0.0993 & 5104.88 & 0.1512 & 0.0935 & 5245.77 & 5104.88 \\
\hline 10 & 0.1558 & 0.1001 & 5104.88 & 0.1513 & 0.0945 & 5200.81 & 5104.88 \\
\hline 15 & 0.1563 & 0.1010 & 5027.83 & 0.1513 & 0.0945 & 5155.60 & 5027.83 \\
\hline 20 & 0.1571 & 0.1020 & 4999.60 & 0.1513 & 0.0950 & 5110.14 & 4999.60 \\
\hline
\end{tabular}




\section{Example-3:}

Let $\mathrm{H}=1$ year $, \alpha=1000, \beta=0.3 ; \mathrm{h}=1.2, \mathrm{P}=20, \mathrm{k}=200 ; \mathrm{S}=30 ; \delta=1 ; \mathrm{I}_{\mathrm{r}}=0.15, \mathrm{I}_{\mathrm{e}}=0.13 ; \theta=0.08, \mathrm{M}=2 / 365 ; \mathrm{i}=0.05 ; \pi=15$; $\mathrm{n}=2$. From the table 3 , if $\mathrm{i}$ increases from $0.05,0.10,0.15,0.20$, the total average cost increases

Table 3: Sensitivity analysis for i:

\begin{tabular}{|c|c|c|c|c|c|c|c|}
\hline \multirow{2}{*}{$\mathrm{I}$} & \multicolumn{3}{|c|}{ CASE-I } & \multicolumn{3}{c|}{ CASE-II } & TVC \\
\cline { 2 - 8 } & $\mathrm{T}^{*}$ & $\mathrm{~T}_{1}{ }^{*}$ & $\mathrm{TVC}_{1}$ & $\mathrm{~T}^{*}$ & $\mathrm{~T}_{1}{ }^{*}$ & $\mathrm{TVC}_{2}$ & $\begin{array}{c}\text { Min }_{\left(\mathrm{TVC}_{1}\right.} \\
\left.\mathrm{TVC}_{2}\right)\end{array}$ \\
\hline 0.05 & 0.1549 & 0.0986 & 5149.07 & 0.05 & 0.1549 & 0.0986 & 5149.07 \\
\hline 0.10 & 0.1538 & 0.0979 & 5187.62 & 0.10 & 0.1538 & 0.0979 & 5187.62 \\
\hline 0.15 & 0.1528 & 0.0972 & 5225.33 & 0.15 & 0.1528 & 0.0972 & 5225.33 \\
\hline 0.20 & 0.1518 & 0.0966 & 5262.27 & 0.20 & 0.1518 & 0.0966 & 5262.27 \\
\hline
\end{tabular}

\section{Example 4:}

Let $\mathrm{H}=1$ year , $\alpha=1000, \beta=0.3 ; \mathrm{h}=1.2, \mathrm{P}=20, \mathrm{k}=200 ; \mathrm{S}=30 ; \delta=1 ; \mathrm{I}_{\mathrm{r}}=0.15, \mathrm{I}_{\mathrm{e}}=0.13 ; \theta=0.08, \mathrm{M}=2 / 365 ; \mathrm{i}=0.05 ; \pi=15$; $\mathrm{n}=2$. From the table 4 , if $\delta$ increases from $1,2,3,4$ the total average cost increases.

Table 4: Sensitivity analysis for $\delta$ :

\begin{tabular}{|c|c|c|c|c|c|c|c|}
\hline$\delta$ & \multicolumn{2}{|l|}{ CASE-I } & \multicolumn{2}{l|}{ CASE-II } & TVC \\
\cline { 2 - 8 } & $\mathrm{T}^{*}$ & $\mathrm{~T}_{1}{ }^{*}$ & $\mathrm{TVC}_{1}$ & $\mathrm{~T}^{*}$ & $\mathrm{~T}_{1}{ }^{*}$ & $\mathrm{TVC}_{2}$ & $\begin{array}{c}\mathrm{Min}( \\
\mathrm{TVC}_{1,} \\
\mathrm{TVC}_{2}\end{array}$ \\
\hline 1 & 0.1554 & 0.0989 & 5133.41 & 1 & 0.1554 & 0.0989 & 5133.41 \\
\hline 2 & 0.1482 & 0.1037 & 5382.84 & 2 & 0.1482 & 0.1037 & 5382.84 \\
\hline 3 & 0.1437 & 0.1070 & 5551.27 & 3 & 0.1437 & 0.1070 & 5551.27 \\
\hline 4 & 0.1406 & 0.1904 & 5672.79 & 4 & 0.1406 & 0.1904 & 5672.79 \\
\hline
\end{tabular}

Graph For sensitivity analysis for $\mathrm{K}$ :

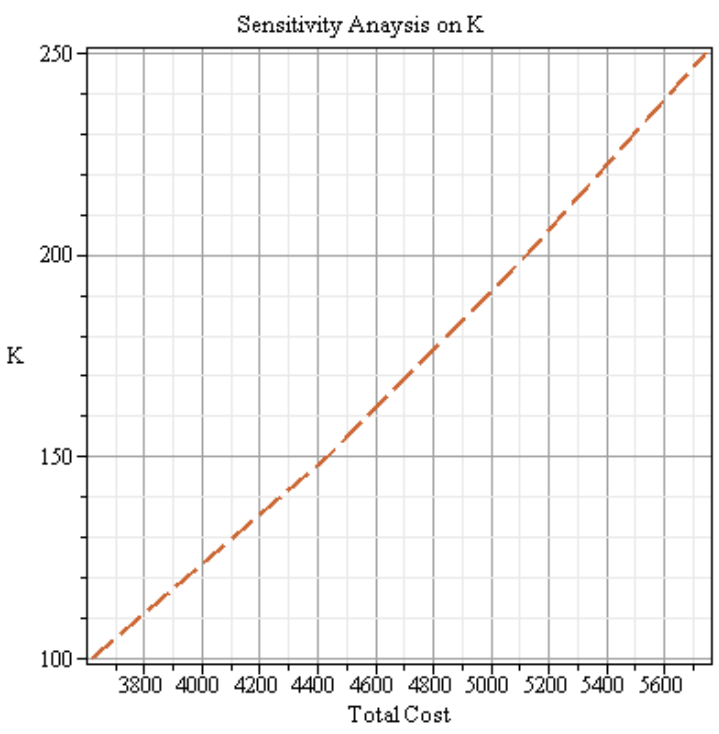


Graph for sensitivity analysis on M:

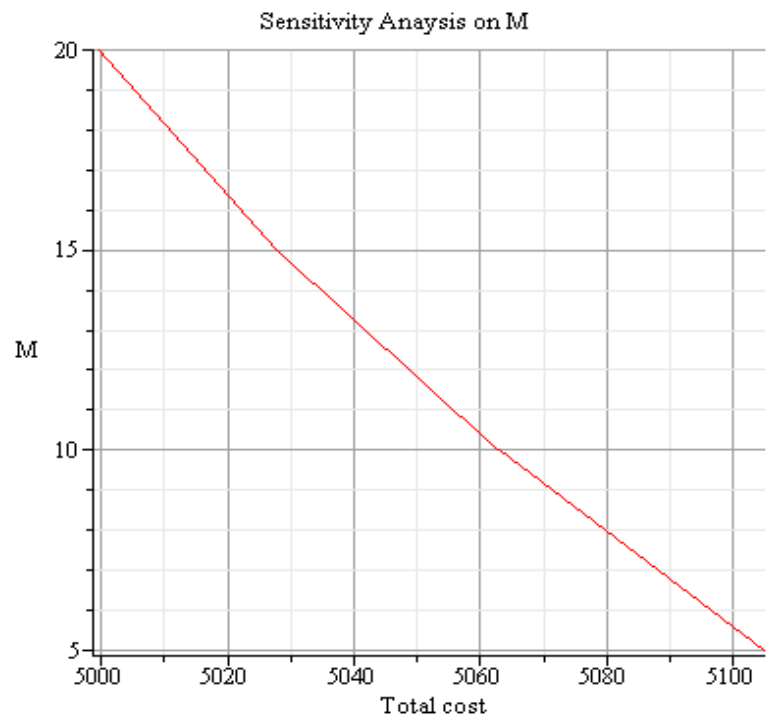

Graph for sensitivity analysis on i:

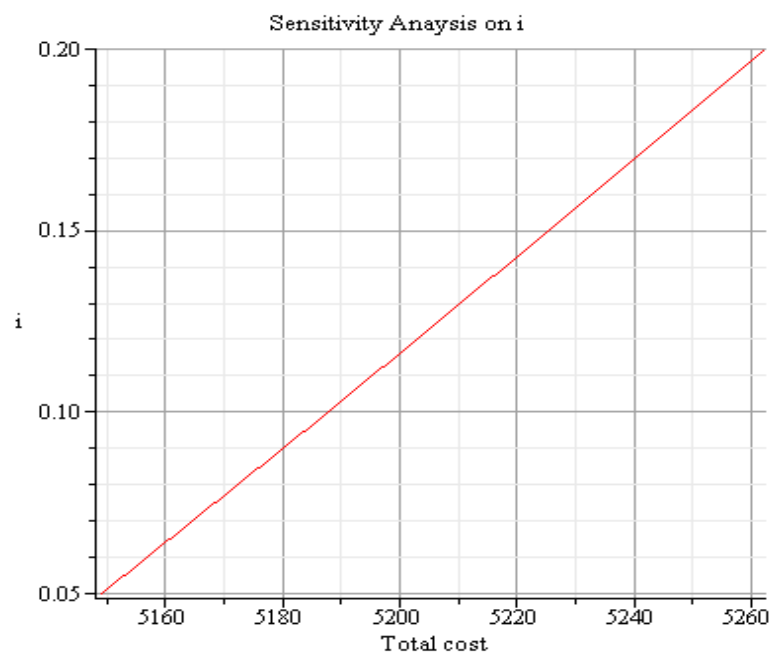

Graph for Sensitivity analysis on $\delta$ :

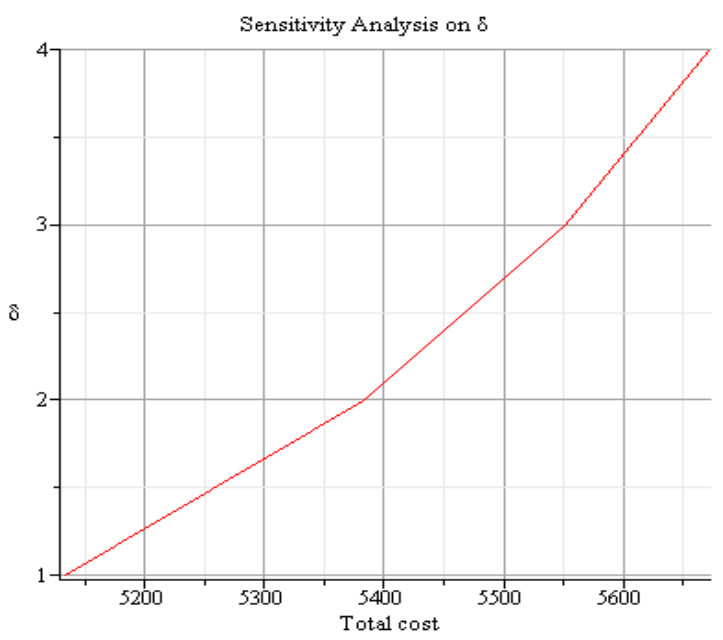




\subsection{Managerial Implications}

Based on the numerical examples considered above, the effects of change of $\mathrm{K}, \mathrm{M}, \delta$, i on the optimal values of the total average cost is studied.

1. In example 1 , different values of $\mathrm{K}$ are considered. The computed results are shown in the Table 1. From these computed results, it is inferred that a higher value of ordering cost $\mathrm{K}$ implies higher total cost. Hence the retailer should lessen the ordering cost.

2. In example 2, the problem with various values of $\mathrm{M}$ are analyzed. Table 2 gives a clear picture that as the credit period increases, the total cost decreases. So the supplier's permissible delay makes the retailer very lucrative i.e., the retailer is most beneficiary if he gets longer permissible delay period from the supplier.

3. In example 3, the effects of inflation rate is studied. The results are given the table 3 . From these results, a higher value of inflation rate implies larger value of the total average cost .

4. In example $4, \mathrm{M}$ and $\mathrm{K}$ are fixed and different values of $\delta$ are considered. The results are given in the table. As the value of the $\delta$ increases the total average cost also increases. It is implied that the retailer should restrict the backlogging parameter with the aim of reducing the average total inventory cost

\section{CONCLUSION}

This paper presents an inventory model of direct application to the business that considers the fact that the storage items are deteriorated during storage periods. The model allows for shortages and the demand is partially backlogged. The objective of our model is to minimize the retailer's total inventory cost through various parameters like permissible delay in payments, inflation rate and ordering cost. The model is solved analytically by minimizing the total inventory cost. Finally the proposed model has been verified by the numerical examples and graphical analysis.

In practical situations, the information about inventory is always precise, most of the time it is vague or imprecise. So it is more reasonable to develop an dynamic research method and is also the future trend of deteriorating inventory study. The model presented in this study provides a basis for several possible extensions. For future research, this model can be extended to accommodate planned shortages, variable costs, different rates of inflation, etc. This model is very practical for the retailers who use the preservation technology for deteriorating items. Sensitivity analysis proves that the total inventory cost can be reduced.

\section{REFERENCES}

[1]. Goyal S.K (1985) Economic Order Quantity under Conditions of Permissible Delay in Payments, Journal of the Operational Research Society Vol.36, 335-338.

[2]. Hu..F \& Liu.D (2010) Optimal Replenishment Policy in Payments with Allowable Shortages, Applied Mathematical modelling 34,3108-3117.

[3]. Huang.Y.F (2007) Economic Order Quantity Under Conditional Permissible Delay in Payments, European Journal of Operational Research ,176,911-924.

[4]. Chang C.T. WV, S.J \& Chen L.C (2009) Optimal Payment Time with Deteriorating Items Under Inflation and International Journal of System Sciences, 40,985-993.

[5]. Chun-Tao Chang (2004) An EOQ model with Deteriorating Items under Inflation When Supplies Credits Linked to Order Quantity, International Journal of Production Economics 88, 307-316.

[6]. Suza Cott (1975) Economic Order Quantity with Inflation, Operational Research Quarterly 26, 553558.

[7]. Hou .K.L \& Lin L.C.(2006) An EOQ Model for Deteriorating Items with Price and Stock Dependent Selling Rates under Inflation and Time Value of Money, International Journal of System Sciences 37, 1131-1139.

[8]. Hou K.L. \& Lin L.C (2006) An Inventory Model for Deteriorating Items with Stock Dependent Consumption Rate and Shortages under the Inflation and Time Discounting, European Journal of Operational Research 168, 463-474.

[9]. Mirzazadeh, A .Syeed Esafahani.M. FatemsiGhomi.M.T (2009) An Inventory Model under Uncertain and Inflationary Conditions, Finite Production Rate, International Journal of System Sciences, 40,21-31. 